\title{
Temporary Epicardial Pacing after Open Heart Surgery in Bangladesh: Complications and Prevention
}

Md. Zulfiker Ali Faruquee ${ }^{1 *}$, Md. Atiqur Rahman², Md. Ibrahim Khalilullah ${ }^{3}$, Md. Shahnoor Aziz ${ }^{4}$, Rokonuzzaman Selim 5 , Syed Tanvir Ahmad ${ }^{6}$, Abdullah al Shoyeb

\author{
${ }^{1}$ Consultant, Associate Professor, Department of Cardiac Anesthesiology, Ibrahim Cardiac Hospital \& Research Institute, Dhaka, \\ Bangladesh \\ ${ }^{2}$ Specialist Cardiac Surgeon, Department of Cardiac Surgery, Ibrahim Cardiac Hospital \& Research Institute, Dhaka, Bangladesh \\ ${ }^{3}$ Assistant Professor, Department of Cardiac Anesthesiology, Ibrahim Cardiac Hospital \& Research Institute, Dhaka, Bangladesh \\ ${ }^{4}$ Specialist Cardiac Surgeon, Department of Cardiac Surgery, Ibrahim Cardiac Hospital \& Research Institute, Dhaka, Bangladesh \\ ${ }^{5}$ Consultant, Associate Professor, Department of Congenital Cardiac Surgery, Ibrahim Cardiac Hospital \& Research Institute, Dhaka, \\ Bangladesh \\ ${ }^{6}$ Assistant Professor, Department of Cardiac Surgery, Ibrahim Cardiac Hospital \& Research Institute, Dhaka, Bangladesh \\ ${ }^{7}$ Specialist Cardiac Surgeon, Department of Cardiac Surgery, Ibrahim Cardiac Hospital \& Research Institute, Dhaka, Bangladesh
}

DOI: $\underline{10.36347 / \text { sasjs.2021.v07i02.020 }}$

| Received: 27.01.2021 | Accepted: 11.02.2021 | Published: 28.02.2021

*Corresponding author: Dr. Md. Zulfiker Ali Faruquee

Background: Epicardial wires allow temporary pacing after cardiac surgery. Pacing is often the best, and sometimes the only method of treating temporary rhythm disturbances in this context. Temporary epicardial pacing has evolved from simple one-chamber systems to dual chamber, biatrial, and even biventricular systems. Temporary wires are routinely sutured to atrial and/or ventricular epimyocardium after open heart surgery. Despite the common problems of poor sensing or capture, dislodgement or retention, no criteria exists for proper placement or removal. This report describes clinical complications due to: failure of ventricular sensing, failure of ventricular capture, bleeding from right ventricular laceration with tamponade. Appropriate placement of temporary wires for optimum function requires 2-cm electrode separation, application into both atrium and ventricle with a gentle redundant loop to exit near the midline, avoiding both coronary arteries and coronary artery bypass grafts. Gentle traction for removal is recommended on the day prior to discharge, especially for patients on antiplatelet or anticoagulant therapy. Aim: Evaluation of complication and to prevent complicatiom in temporary epicardial pacing after open heart Surgery in Bangladesh. Method: This observational descriptive study was conducted in Ibrahim Cardiac Hospital \& Research Institute, Dhaka, Bangladesh on 200 patients received temporary epicardial pacing treatment during the period from January 2018 to December 2019. Result: The study population was 200. The highest 123(61.5\%) were age group between 60 years to 69 years. The study found 126(63.0\%) were Male and 74(37.0\%) were Female. The study population of postoperative complications of temporary epicardial pacing open heart surgery was 20. The highest 11(55.0\%) were Failure of ventricular sensing with resultant VT/VF. Conclusion: Epicardial pacing set and check properly at the right time and beware while opening it. We hope there will be no problem of temporary Epicardial Pacing for patients.

Keywords: Epicardial pacing, Cardiac Surgery, Aortic valve replacement (AVR).

Copyright (C) 2021 The Author(s): This is an open-access article distributed under the terms of the Creative Commons Attribution 4.0 International License (CC BY-NC 4.0) which permits unrestricted use, distribution, and reproduction in any medium for non-commercial use provided the original author and source are credited.

\section{INTRODUCTION}

Epicardial wires allow temporary pacing after cardiac surgery. Pacing is often the best, and sometimes the only method of treating temporary rhythm disturbances in this context. Temporary epicardial pacing has evolved from simple one-chamber systems to dual chamber, biatrial, and even biventricular systems [1]. Common indications for permanent pacing after cardiac surgery include complete heart block, sinus node dysfunction (sick sinus syndrome and other tachy-brady syndromes), slow ventricular response to atrial fibrillation, and second degree Mobitz type II heart block with an inadequate ventricular rate [2]. Complications of epicardial wires include infection, myocardial damage, ventricular arrhythmias, perforation, and tamponade [3, 4]. After cardiac surgery, temporary pacing leads are routinely implanted in the right ventricle (RV). Temporary wires are sutured routinely to the atrial and/or ventricular epimyocardium 
after open heart surgery. These wires are of value for the treatment of cardiac arrhythmias following surgery, and by the maintenance of appropriate rate or atrioventricular sequence, may facilitate improved cardiac output [5-7]. Despite the common problems of poor sensing or capture (intermittent, erratic or absent), dislodgement, and occasional retention, no criteria exists for proper placement or removal of these temporary epimyocardial wires. Temporary epicardial pacemakers have become increasingly sophisticated over the years and have evolved from simple single chamber devices with few programmable parameters to complex dual chamber devices capable of adjustable parameters similar to permanent pacemakers. This study will describe the various indications for temporary pacing, technical considerations for both the choice of pacing wires and pacemaker modes, complications with temporary pacing and our current practice with temporary pacing [8]. Figure 1 and 2 were taken from internet.

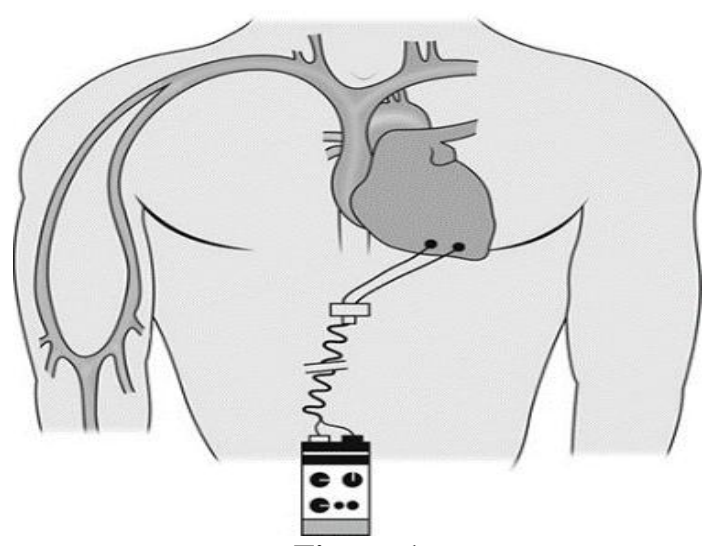

Figure-1

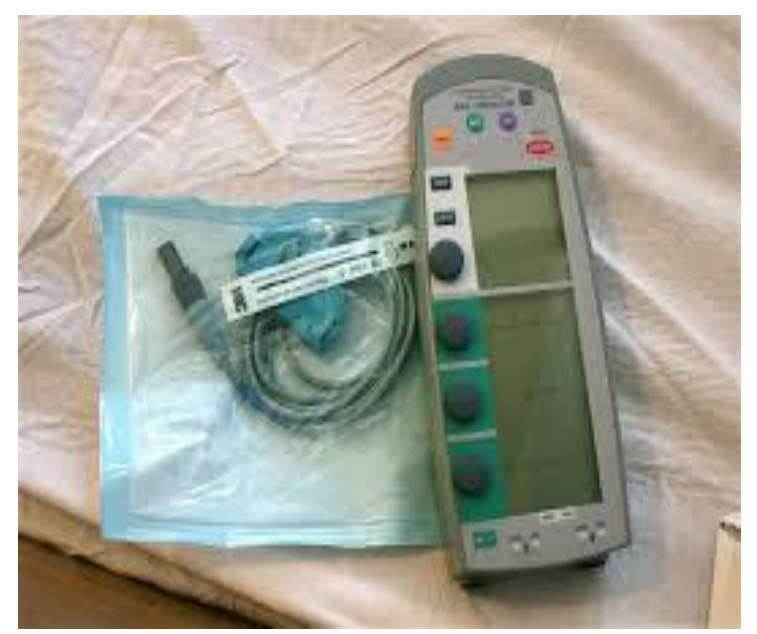

Figure-2

Epicardial wires allow temporary pacing after cardiac surgery that are attached to a pacer box via a pacing cable. It is given when the patients own intrinsic or built in ability to pace fails or to cause a more effective depolarization. Pacing is often the best, and sometimes the only method of treating temporary rhythm disturbances in this context. Temporary epicardial pacing is indicated during-Any slow rate where the patient is symptomatic, like-Sinus bradycardia, $2^{\text {nd }}$ and $3^{\text {rd }}$ degree heart block, junctional rhythm, idioventricular rhythm. Temporary epicardial pacing leads are typically placed in the operating room after the cardiac procedure is completed and before chest closure. Epicardial pacing wires were historically placed only on the right ventricle, can given in right atrium also. This allowed for management of severe bradyarrhythmias with a more stable ventricular rate but at the expense of AV synchrony. An implanted pacing lead forms a direct current path to the myocardium. During pacing lead insertion and testing, only batterypowered equipment specially designed for this purpose should be used to protect against fibrillation that may be caused by alternating currents. Virtually all patients at most cardiac surgical centers received temporary epicardial pacing wires (TEPW). Although the incidence of complications from placement or removal of TEPW has been low, the adverse events can cause major morbidity and even mortality. In addition to inade-quate lead function, the majority of serious morbidity reported relates to lead removal. Complications of epicardial wires include failure of ventricular sensing with resultant VT/VF, Failure of ventricular capture, Bleeding from pacemaker wire sites, Right ventricular laceration with bleeding and tamponade.

\section{OBJECTIVE}

Evaluation of temporary epicardial pacing after open heart Surgery in Bangladesh. Assessment of problem and solution in it.

\section{METHODOLOGY \& MATERIALS}

This observational descriptive study was conducted in Ibrahim Cardiac Hospital \& Research Institute, Dhaka, Bangladesh during the period from January 2018 to December 2019. The study was approved by the ethical committee of the mentioned hospital. Proper written consents were taken from all the participants before starting the main part of intervention. Obeying inclusion and exclusion criteria of this study in total 200 cardiac surgery patients underwent the temporary epicardial pacing procedure were selected as study population for analyzing data. According to the exclusion criteria of the study, data of severely ill patients and data of patients with other three or more complex diseases like diabetes mellitus, hepatitis, pyelonephritis, cancer etc. were excluded. All necessary data were collected, analyzed and disseminated by several program of MS-Office and SPSS version 22 .

\section{RESULT}

The study population was 200. The highest $123(61.5 \%)$ were age group between 60 years to 69 years followed by aged 50 years to $>70$ years, 
$69(34.5 \%)$ were 50 years to 59 years, $123(61.5 \%)$ were 60 years to 69 years, $8(4.0 \%)$ were $>70$ years. The study found $126(63.0 \%)$ were Male and $74(37.0 \%)$ were Female. The study population of postoperative complications of temporary epicardial pacing open heart surgery was 20 . The highest $11(55.0 \%)$ were Failure of ventricular sensing with resultant VT/VF, $5(25.0 \%)$ were Failure of ventricular capture, 2(10.0\%) were Bleeding from pacemaker wire sites and $2(10.0 \%)$ right ventricular laceration with bleeding. Figure-2 demonstrated the Distribution of study patients according to BMI.

Table-1: Distribution of study population according to

\begin{tabular}{|l|l|l|} 
Age $(\mathbf{n = 2 0 0})$ \\
\hline Age group (years) & $\mathbf{n = 2 0 0}$ & $\mathbf{\%}$ \\
\hline 50 years to 59 years & 69 & 34.5 \\
\hline 60 years to 69 years & 123 & 61.5 \\
\hline$>70$ years & 8 & 4.0 \\
\hline Total & 200 & 100.0 \\
\hline
\end{tabular}

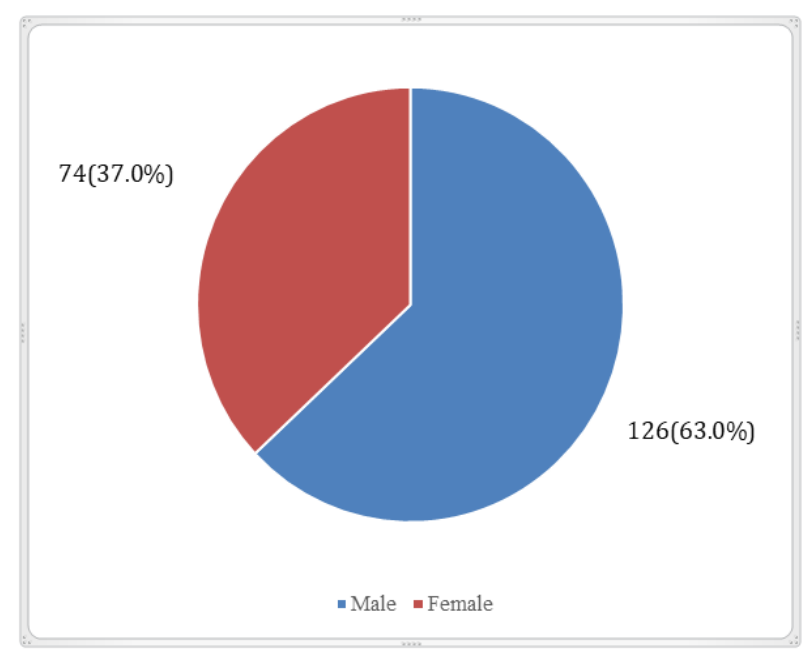

Fig-3: Distribution of study population according to $\operatorname{Sex}(n=200)$

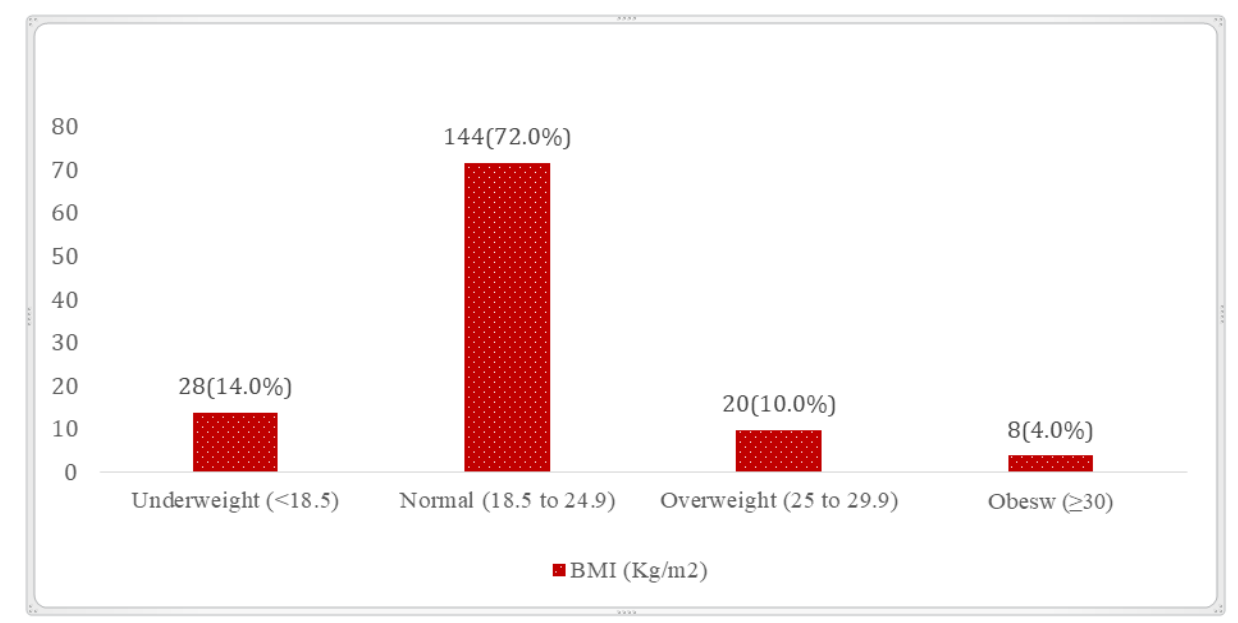

Fig-4: Distribution of study population according to BMI $(n=200)$

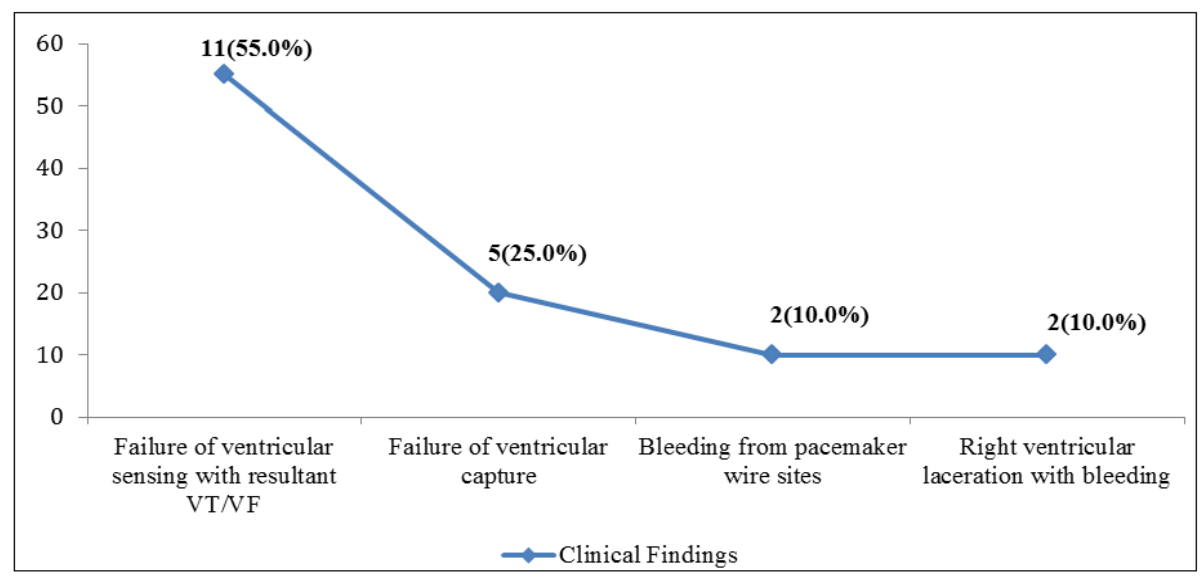

Fig-5: Postoperative Complications of Temporary Epicardial pacing after open heart surgery

\section{DISCUSSION}

This study included 11 patients who developed failure of ventricular sensing requiring temporary pacing (VVI mode) for intermittent AV block among of them in 4 CABG, 4 MVR and 3 AVR. Poor sensing of intermittent spontaneous beats was noted but not treated. Later on, ventricular tachycardia developed from pacer induced VPB's that deteriorated to ventricular fibrillation requiring countershock. Among them, one patient need insertion of a temporary transvenous electrode, the patient later underwent uneventful insertion of a permanent cardiac pacemaker. 
This study included 5 patients developed failure of ventricular capture of them in $2 \mathrm{CABG}, 3 \mathrm{MVR}$. Because of chronic atrial fibrillation, only two ventricular wires were inserted at surgery but were noted not to be capturing in the ICU. Thresholds for stimulation had not been tested in the operating room. Ventricular bradycardia ensued with low cardiac output and the rate was unresponsive to increasing inotropes. A temporary transvenous electrode was inserted all of these case via the right subclavian vein and augmentation of ventricular rate immediately restored cardiac output. All patients postoperative course were uneventful. This study included 2 patients developed bleeding from pacemaker wire site underwent mitral valve replacement: two pacer wires were left in each of the right atrium and right ventricle. In one case brisk postoperative bleeding $(400 \mathrm{cc} / \mathrm{h})$ prompted reexploration 3 hours later and in another case reexploration done after 5 hours and found $200 \mathrm{cc} / \mathrm{hr}$ postoperative bleeding. Both cases the bleeding was identified at the insertion site of one of the right ventricular pacer wires. The wire had been inserted through the full thickness of the right ventricle. Wires was removed, resutured and the bleeding stopped. The wire was reapplied more superficially at another location. This study included 2 patients developed right ventricular laceration with bleeding and tamponade underwent aortic valve replacement and was started on Coumadin after initial heparinization for 3 days. The prothrombin time was in the therapeutic range. Few minutes after their removal, the patient fainted and was markedly hypotensive. Cardiac arrest required openchest massage on the ward; acute pericardial tamponade due to right ventricular laceration was noted at the site of wire removal. Both patient recovered, did not develop sternal infection, and was discharged 14, 15 days later respectably. Appropriate placement of temporary wires for optimum functioning requires at least $2 \mathrm{~cm}$ separation and adequate contact with myocardium, not just epicardium. Figure-1 illustrates application of the ventricular wires in the outflow tract of the right ventricle in an area devoid of coronary arteries, sufficiently deep to ensure myocardial contact, but not so deep as to enter the ventricular chamber. We had utilized a U-shaped insertion, but now ensure that a straight bite is taken. Often, one ventricular wire is placed on the inflow tract or on the inferior RV surface. The direction of electrode insertion is to allow easy removal by simple, gentle traction inferiorly. A fixation stitch of 5-0 silk is rarely required. Regarding the atrium, one wire is inserted in the thick wall near the interatrial groove and one through the remnant of atrial wall at the ligated venous drainage tourniquet site. We try not to suture the wires in place, ensuring that the ends are cut clean without fraying and that there is an adequate redundant loop in the pericardial cavity that allows for moving the heart about to inspect distal anastomoses or reopening the chest. We ensure that no saphenous vein bypass graft lies in proximity to any bare wire, and especially a right coronary bypass graft with hemoclips on side branches. Care is taken in the placement of mediastinal and pericardial chest tubes so that the temporary wires are not looped about the catheters. The wires are inserted prior to protamine administration to ensure hemostasis and then checked prior to sternal closure, looking for either bleeding or dislodgement (which is not uncommon for the atrial wires). The wires may be loosely sutured to the pericardium to ensure that they do not loop around the drains. The wires are then sutured to the skin in a circular manner that allows feeding extra length, should reopening for massage or bleeding be required. The wires are brought out a few centimeters from either side of the linea alba usually just superior to the pericardial and mediastinal drains. After bringing out the Keith needles through the skin, the distal IMA and superior epigastric area are checked for hematoma or bleeding. Thresholds for pacing and sensing should be performed in the operating theater, prior to chest closure and should be reassessed in the ICU after initial stabilization of the patient. Failure to do so may result in in appropriate sensing or pacing at a critical time. Transvenous or transthoracic pacing systems may be employed in emergency situations should thresholds on the temporary wires be inadequate.

Epicardial pacemaker wires are a low resistance connection to the heart. This creates the potential for microshock- induced arrhythmia, particularly ventricular fibrillation. Patients must be nursed in a cardiac- protected electrical environment, which implies adequately isolated electrical equipment and measures to prevent build- up of static electricity (absence of carpet, for example). Wires should only be handled with non- conductive gloves, and a large metal object (the bed, for example) should be touched first to discharge static potential prior to touching the wires. The wires should be protected in a non- conductive container (for example, a plastic syringe barrel) when not in use [2]. To minimise unwanted electrical interference, by default, modern digital ECG monitors apply a high frequency filter to the incoming signal. In most cases this filters out the brief pacemaker spike, making it difficult to tell whether a pacing stimulus is being delivered. This can be overcome on most modern monitors by selecting the 'pacemaker' mode, which will record each spike, often highlighted with a marker. The small skin potentials created by bipolar leads in particular may be difficult to detect, and it may be necessary to inspect a variety of leads. Extracardiac, high frequency interference may become problematic, in which case the monitor can be returned to its usual mode after pacing diagnostics have occurred ${ }^{2}$. Electrical pacemaker output does not necessarily equate to mechanical capture of the myocardium, and as such it is helpful to have a monitor demonstrating the timing of cardiac contraction. An arterial pressure tracing or pulse oximeter waveform are the most readily available, and can usually be printed next to the ECG on a cardiac rhythm strip. This is much more helpful than attempts at 
Zulfiker Ali Faruquee et al., SAS J Surg, Feb, 2021; 7(2): 107-111

analysing the moving screen image. If attempting to find pacing settings that produce optimal cardiac output, it is also helpful to have a high time- resolution monitor of cardiac output, such as echocardiography, continuous cardiac output pulmonary artery catheter, mixed venous oxygen, or one of the pulse contour analysis devices. Pacemaker- dependent patients are at risk of pacemaker system failure or pacemaker- generated arrhythmia, and as a minimum should have continuous ECG monitoring and immediate access to a cardiac defibrillator with the capacity for transcutaneous pacing [9]. Pacing wires should be removed only after therapeutic heparin has been discontinued and before warfarin is commenced. They are removed by constant gentle traction, allowing the motion of the heart to assist dislodgement from the epicardial surface. Excessive traction should not be applied: occasionally the wires are caught by a tight suture either in the epicardium or somewhere along their course through the chest. In this case they should be pulled as far as is felt safe, and cut as close to the skin as possible. This will allow the cut ends to retract. There is no evidence that wires left like this have any adverse effect. Care should be taken to avoid the possibility of unintentional contact between the pacing lead(s), including extension cable, and any equipment used as well as any conductive surface contact. While temporary pacing leads are removed by gentle traction, ECG should be monitored (observing for arrhythmias) and the patient should be observed for a few hours after lead removal because of the risk of tamponade. Leaving the lead implanted for longer than 7 days may result in difficulty or inability to extract the lead and/or bleeding.

\section{CONCLUSION}

Epicardial pacing set and check properly at the right time and beware while opening it. We hope there will be no problem of temporary Epicardial Pacing for patients.

\section{REFERENCES}

1. Reade MC. Temporary epicardial pacing after cardiac surgery: a practical review: part 1: general considerations in the management of epicardial pacing. Anaesthesia. 2007 Mar;62(3):264-71.

2. Reade MC. Temporary epicardial pacing after cardiac surgery: a practical review. Part 2: Selection of epicardial pacing modes and troubleshooting. Anaesthesia. 2007;62(4):364373.

3. Carroll KC, Reeves LM, Ray GA. Risks associated with removal of ventricular epicardial pacing wires after cardiac surgery. American Journal of Critical Care. 1998 Nov 1;7(6):444-9.

4. Reade MC. Temporary epicardial pacing after cardiac surgery: A practical review: part 1: General considerations in the management of epicardial pacing. Anaesthesia. 2007;62:264-71.

5. Mills NL, Ochsner JL. Experience with atrial pacemaker wires implanted during cardiac operations. J Thorac Cardiovasc Surg. 1973;66(6):878-886.

6. Waldo AL, MacLean WA, Cooper TB, Kouchoukos NT, Karp RB. Use of temporarily placed epicardial atrial wire electrodes for the diagnosis and treatment of cardiac arrhythmias following open-heart surgery. J Thorac Cardiovasc Surg. 1978;76(4):500-505.

7. Hartzler GO, Maloney JD, Curtis JJ, Barnhorst DA. Hemodynamic benefits of atrioventricular sequential pacing after cardiac surgery. Am J Cardiol. 1977;40(2):232-236.

8. Batra AS, Balaji S. Post operative temporary epicardial pacing: When, how and why?. Annals of pediatric cardiology. $2008 \mathrm{Jul} ; 1(2): 120-125$.

9. Health Quality Ontario. Remote monitoring of implantable cardioverter-defibrillators, cardiac resynchronization therapy and permanent pacemakers: a health technology assessment. Ontario health technology assessment series. 2018;18(7):1-199.

10. Hilton JD, Weisel RD, Baird RJ, Goldman BS, Jablonsky GE, Pym JO, Scully HE, Ivanov JO, Mickle DA, Feiglin DH, Morch JE. The hemodynamic and metabolic response to pacing after aortocoronary bypass. Circulation. 1981 Aug 1;64(2 Pt 2):II48-53.

11. Goldman B, Hilton D, Weisel R, Ivanov J, Pym J, Jablonsky G, Mclaughlin P, Feglin D. Atrial vs ventricular pacing in compliant and noncompliant hearts: effects on cardiac performance and metabolism. PACE, 1981; 4: A-48.

12. Peters RW, Scheinman MM, Raskin S, Thomas AN. Unusual complications of epicardial pacemakers. Recurrent pericarditis, cardiac tamponade and pericardial constriction. Am J Cardiol. 1980;45(5):1088-1094.

13. Breivik K, Engedal H, Resch F, Segadal L, Ohm OJ. Clinical and electrophysiological properties of a new temporary pacemaker lead after open-heart surgery. Pacing Clin Electrophysiol. 1982;5(4):600-606.

14. Morin JE, Wynands JE, Ralphs-Thibodeau S. Temporary cardiac pacing following open-heart surgery. Can J Surg. 1982;25(2):128-131.

15. Parsonnet V, Bhatti M. A technique for postoperative application of a newly designed temporary bipolar dual-chamber pacemaker electrode. J Thorac Cardiovasc Surg. 1985;89(3):456-458.

16. Breivik K, Engedal H, Resch F, Segadal L, Ohm OJ. Clinical and electrophysiological properties of a new temporary pacemaker lead after open-heart surgery. Pacing Clin Electrophysiol. 1982;5(4):600-606.

17. Aris A, Cámara ML, Padró JM, Oriol Bonnin J, Oriol Solé J, Caralps JM. Clinical evaluation of a new temporary pacemaker wire. Ann Thorac Surg. $1983 ; 36(2): 228-230$. 